"Vox Patrum" 6/1986/ z.11

\title{
CHARISMA VERITATIS CERTUM (Adversus haereses IV 26,2)*
}

C'est dans la deuxieme partie ${ }^{1}$ du Livre IV de l'Adversus haereses sur"l'Ancien Testament, prophét1e du Nouveau" que se trouve la section ${ }^{2}$ sur l'enselgnement des presbytres commençant par l'affirmation d'Irénée de Lyon que les presbytres ont regu le "sûr charisme de vérité" /charisma veritatis certum/:

"C'est pourquoi, $11-11,11$ faut écouter les presbytres qui sont dans l'Eglise: 118 sont les successeurs des apôtres, ains1 que nous 1 'avons montré, et, ayec la succession dans l'épiscopat, 11 s ont regu le sûr charisme de vérité, selon le bon plaisir du Pere"

Cette affirmation d'Irénée a été reprise par le Concile de Vatican II, dans la constitution "Del Verbum", à propos de la Tradition apostollque qui progresse dans l'Église grâce à la "prédication de ceux qui, avec la succession dans l'épiscopat, ont regu un charisme qui certifie la vérité" 5 .

* La conférence prononcée pondant le Colloque d'llistoire Ecclésiastique comparée sur "L'Insiltution et les pouvolrs dans les Eglises de I'Antiquité à nos jours", Strasbourg 21-24 septembre 1983.

1 Adversus haereses IV 20-35, SCh 100, 624-877.

2 Adversus haereses IV 26,2-32,2, SCh 100, 718-800.

3 En Adversus haereses III 2,1 et $3,1 / \mathrm{SCh} 211,24-26$ et $30 /$ Irénée a montré que les presbytres sont les évêques dés différentes églises locales qui conservent la tradition des Apotres. Voir l'interprétation du passage Adversus haereses III $3,-1-3$ donnée par A. liousseau in SCh 210, 223-236.

4 Adversus haereses IV 26,2, SCh 100,719.

5 "... ex praeconio eorum qui cum episcopatus successione charisma veritatis certum acceperunt". De1 Verbum II, 8. La Révélation divine, t.1: Constitution dogmatique "De1 Verbum", texte latin et traduction Prançaise de J.P. Torrell, Paris 1968, 30. Voir la commentalre qu'en donne Umberto Bett1, i'un les rédacteurs de ce texte, dans son article "Le magigtere de l'Eglise au service de la Parole de Dieu: a propos du n 10 de la Constitution dogmatique 
- Mais que signifie exactement l'expression "charisas veritat1s certum" dans l'Adversus haereses?

- ot quel rapport Irénée établit-1l entre charisme et vérité, de telle sorte qu'on puisse parler d'un "sûr charisme de la véritén?

C'est 'ces deux questions que je veux répondre sachant que toute interprétation de ce texte est porteuse d'une ecclésiologie différente et que l'enjeu du débat est celui du nmagietèrén épisoopai ${ }^{6}$.

1. Tout d'abord comment Irénée définit-1l les charismes et quels sont-11s?

Le terme "char1sme" $/ \chi d \rho \iota \sigma \mu \alpha / 7$ se renoontre 11 fols dans l'Adversus haereses dont 8 au Livre IV. Dans tro1s cas, 11 s'agit de

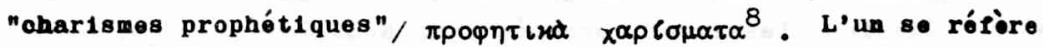
aux prophètes de l'A.T., qui ont reģu du Verbe un "ohar1sme prophót1que": "Ils ont prôché à l'avance sa venue selon la cha1r par laquelle le mélange et la communion de Dieu et de l'home ont été ré-

"De1 Verbum", In: Au Service de la Parole de Dieu. Mólanges ofPerts à Mgr A.M. Charue, Gembloux 1969, 245-261: "Les éveques, dit-1l, en tant qu'1ls participent aux pouvo1rs du Christ selon la plénitude sacramentelle, sont les seuls depositaires d'un charisme sur de vérité. Ce charisme sacramentel les habilite à juger on derniére instance de l'authenticité et de l'usago ordonné des charismes, d'origine autre que sacramętelle, lesquels peurent se trouvęr chez les lalcs ou chez les prétres ou chez les évéques eux-memes. C'est leur devo1r de ne pas éteindre l'Esprit; ma1s c'est aussi leur devoir de tout éprouver afin de rotenir seulement ce qui est bon", /p.253/.

6 Irénée parle de l'épiscopat comme "locus magisteri1"/III 3,1/ qui a pour fonction l' "administratio sermonis" $/ V, P r$, comparer à IV 26,4\%, Cependant à la suite du Pore Yves Congar, "Pour une h1sto1re semantique du terme "Magisterium" et "Breve histoire des Pormes du "Mag1steren et de 808 relations avec les docteurs", "Revue des sciences philosophiques et thóolog1ques" 60/1976/, 85-98 et 99-112, nous parlerons de "mission d'enselgnement" plutôt que de "magistere". Volr aussi John P. Boyle, "The ordinary Magisterlum: towards a history of the concept", "The Heythrop Journal" $20 / 1979 / 380-398$ et 21/1980/ 14-29.

7 "Charisma" ( $\chi d \rho\llcorner\sigma \mu \alpha$ ) se trouve en Adversus haereses III $11,9, \mathrm{SCh}$ 211,172 ; IV 20,4 et $6 / 2$ fo1s/, $7 ; 26,2$ et $5 ; 27,2 ; 33,8$, SCh 100 $634,642,646,718,728,740,820 ; v^{\prime} 6,1 ; 22,1$, SCh 153,74,280.

8 Cer Adversus haereses III $11,9, \operatorname{SCh} 211,172 ; \operatorname{IV} 20,4, \operatorname{SCh} 100,634$; 
alisés selon le bon plaisir du Père/secundum placitum Patris/" ${ }^{9}$. Les deux autres se réfèrent à la $I^{e ̀ r e ~ E p i ̂ t r e ~ a u x ~ C o r i n t h i e n s, ~}$ où Paul "a parlé avec précision des charismes prophétiques car 11 connâ̂t des hommes et des fenmes qui prophétisent dans l'Eglise/1n Ecclesia/n10, et encore: "nombre de frères dans l'Eglise / In Ecclesia/ qui possèdent des charismes prophétiques, parlent toutes sortes de langues grâce à l'Esprit, manifestent les secrets des hommes pour leur profit et exposent les mystères de Dieu"11. C'est l'Eglise qu1 a regu la "grâce prophétique" par "le don de l’Esprit/donum Spiritus/ répandu aux derniers temps sur le genre humain selon le bon plaisir du Père/secundum placitum Patris/"12.

De même c'est "dans l'Eglise/in Ecclesia/n"13 et "selon le bon plaisir du Père/secundum placitum Patris/" que les presbytres ont regu le "sûr charisme de vérité".

Enfin Irénée parle du "don suréminent de la charité/praecipuum dilectionis munus/" dans un texte où il replace la doctrine des charismes dans la perspective de la connaissance de la vérité et de l'apostollcité de l'Eglise:

"La gnose vraie est la doctrine des apôtres; l'antique organisme de l'Eglise répandu dans le monde entier; la marque distinctive du Corps du Christ consistant dans les successions des évêques, auxquels ceux-là remirent chaque église locale; parvenant jusqu'à nous une conservation sans feinte des Ecritures, un compte intégral de celles-c1... enfin le don suréminent de la charité, plus précieux que la "gnose", plus glorieux que la prophétie, supérieur à tous les autres charismes"14.

9 Adversus haereses IV 20,4, SCh 100,635.

10 Adversus haereses III 11,9, SCh $211,173$.

11 Adversus haereses $V 6,1, \operatorname{SCh} 153,75$.

12 Adversus haereses III 11,9, SCh 211,171-173.

13 L'emploi de "In" avec l'ablatif dans l'expression "in ecclesia" apparait en: III 4,$1 ; 11,1 ; 24,1, \operatorname{SCh} 211,46,140,472$; IV 20,12 , $\operatorname{SCh} 100,670 ; V 6,1, \operatorname{SCh} 153,74$. Il ind 1 que 1 'Eglise comme ileu où s'effectue l'action de Dieu, du Christ, de l'Esprit ou des apotres, tandis que "In" avec i'accusatif "in ecclesiam" indique plutôt l'Eglise comme terme de l'action de l'Esprit ou des apotres/cP. III 4, 1 et 11,8, SCh 211, 46 et 164/. "Ecclesia" est aussi employé avec les prepositions "ad" en: III 24,1, SCh 211, 472; V Pr et V 20,2, SCh 153, 12 et 258; "ab" on V Pr, SCh 153, 12; "extra" en IV 33,7 , SCh $100,816$.

14 Adversus haereses IV 33,8, SCh 100, 819-821. 
L'Eglise se caractérise donc par son apostolicité, sa cathol1cité: elle est répandu dans le monde entier; son organicité: elle est un organisme, un $\sigma \delta \sigma \tau \eta \mu \alpha$, ce qui signifie que chaque organe a sa spécificité, mais aussi que tous les membres sont liés au corps de telle sorte que ce qu'un membre falt, c'est le corps tout entier qui la falt, et par le "caractère propre du corps du Christ" qu'est la succession des évêques dans les églises locales.

A cette succession épiscopale est liée la garde ou la conservation non feinte /custoditio sine fictione/ des Ecritures, le compte Intégral /tractatio/ du texte sans addition, ni soustraction, la lectio sine falsatione, la lecture exempte de fraude, et l'expositio legitima et diligens ${ }^{15}$, l'explication correcte et harmonieuse des Ecritures.

Enfin l'Eglise a regu le don de la charité qui est "un don supérieur à tous les autres charismes", o'est pourquol "dans son amour pour Dieu, elle envole en tout lieu et en tout temps une multitude de martyrs au-devant du Père" ${ }^{16}$. Le signe du plus grand amour est le martyre.

2. Or tous ces charismes de Dieu se trouvent, comme nous l'avons dit, dans l'Eglise/1n Ecclesia/:

"Paul enselgne le lieu où on les trouvera: "Dieu dit-11, a établi dans l'Eglise, premièrement 108 apótres, deuxièmement les prophetes, troisiemement los docteurs" 17 .C'est là où furent déposés les charismes de Dieu, qu'il faut s'instruire de la vérité, c'est-a-diro auprès de ceux en qui se trouvent réunies la succession dans l'Eglise depuis les apotres, l'intégrité inat taquable de la conduite,/sanum et irreprobabile conversationis/ et la pureté incorruptible de la parole /et inadulteratum et incorruptibile sermonis/. /Ces hommes là gardent notre fol/fidem nostram custodiunt/ au seul Dieu qui a créé toutes choses; 11 s font croitre notre amour/dilectionem adaugent/envers le Fils de Dieu qui a accompli pour nous de si grandes "économies"; enfin 11 nous expliquent les Ecritures

15 Il y a une gradation entre la tractatio, qui est la conservation intégrale du texte, la lectio sa lecture publique et l'expositio son commentaire ou son interprétation.

16 Adversus haereses IV 33,9, SCh 100, 821.

17 I Cor $12,28$. 
en toute sûreté /et Scripturas... nobis exponunt/, sans blasphémer Dieu, sans outrager les patriarches, sans mépriser les prophètes" 18 .

Là où est l'Eglise, là se trouvent les apôtres, les prophètes

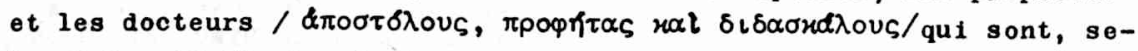
Ion I Cor 12,28, les trois premiers charismes de l'Esprit, donnés en

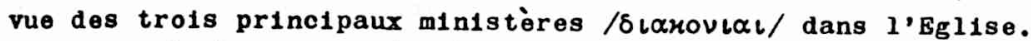

Et là où furent déposès les charismes de bieu, c'est là qu'il faut s'instruire de la vérité, c'est-à-dire auprès de ceux qui /apud quos/ sont les successeurs des apôtres, et possèdent une "conduite Intègre et irréprochable" /sanum et irreprobabile oonversationis/ et "une parole pure et incorruptible /et inadulteratum et incorruptibile sermonis/n 19 , selon la recommandation des Epîtres pastorales/20. Seuls de tels pasteurs 1rréprochables gardent notre fol dans le Créateur, font croître notre amour dans le Fils de Dieu et nous expliquent les Ecritures.

Or cette référence à I Cor 12,28 et à la doctrine paulinienne des charismes 21 se retrouve dans le fameux texte sur l'Eglise et l'Esprit:

18 Adversus haereses IV 26,5, SCh 100, 729.

19 Ib1dem. En IV 26,4/SCh 100, 722-726/Irénée dit qu'on do1t "s'attacher à ceux qui gardent la succession des apôtres et, avec le rang presbytéral, offrent une parole salne/sermonem sanum/ et une conduite Irréprochable /et conversationem sine offensa/". Le "sermo sanus" et la "conversatio sine offensa" sont les deux traits distinctifs des successeurs des apotres. Voir aussi III $3,1, \operatorname{SCh} 211,31$ : "les apôtres voulurent que ceux qu'1ls laissaient comme successeurs fussent très parfaits et frréprochables en tout point".

20 Allusion aux recommandations de saint Paul à Tite: "Mfontre en ta personnè un modèle de belles oeuvres: pureté de doctrine, dignité, parole saine et inattaquable..." /Tt 2,7-8/, et a Tímothê: "Sois pour les fldèles un modele en parole, en conduite, en amour, en Pol, en pureté" $/ 1 \mathrm{Tm} 4,12 \%$

21 Sur la doctrine paulinienne des charismes, volr l'étude de Max-Allain Chevallier, Esprit de Dieu, paroles d'hommes, Neuchâtel 1966, 139-17,1, et sur la doctrine lrénténne l'étude de A. Mehat, Salnt Irénee et les charismes, "Studia Patristica" 18/1982/719-724. 
"Dans 1'Eglise, 'Dieu a placé des apôtres, des prophetes, des docteurs 21', et tout le reste de l'opération de l'Esprit. De cet Esprit s'excluent donc tous ceux qui ${ }_{2}$ refusant d'accourir à l'Eglise, se privent eux-mémes de la vie par lours doctrines lausses et lours actions dépravées ${ }^{22}$. Car là où est l'Eglise, là est auss1 l'Esprit de Dieu; et là où est l'Esprit de Dieu, là est l'Eglise et toute grâce. Et l'Esprit est vérité"23.

Nous trouvons dans ces deux textes /III 24,1 et IV 26,5/ 1a structure fondamentale du ralsonnement d'Irénée qui est une structure Inclus1ve: 'Ub1 - Ibi', 'Là où-là' :

III 24, 1:

Ub1 enim Ecclesia

Ibi et Spiritus Dei

Ub1 Sp1ritus Dei

1111c Ecclesia et omnis gratia

Spiritus autem veritas.

IV 26, 5 :

Ub1 1gitur charismata De1 posita sunt

Ib1 discere oportet veritatem

Là où est l'Eglise, là est l'Esprit et la présence de l'Esprit dans l'Eglise est mantestée par les charismes des membres de l'Egl1se, charismes d'apostolat, de prophétie et d'enseignement - de didascalia - qui sont dûs à l'"opération de l'Esprit" en elle. Car les charismes sont les signes et les fruits de la "vivification" des membres du Corps du Christ par l'Esprit qui est la vie de l'Eglise comme l'ame est la vie du corps. Les charismes sont donnés à certaIns, par l'Esprit, comme des talents ${ }^{24}$, pour remplir une fonction ou jouer un certain rôle dans l'Eglise.

Et là où les charlames de Dieu furent déposés, c'est là qu'il faut s'instruire de la vérité. La relation entre la présence des charismes de l'Esprit dans l'Eglise et l'enseignement de la verité se

22 La "doctrine fausse" et les "actions dépravées" sont les deux caractéristiques des docteurs hérétiques par opposition aux presbytres de l'Eglise qui ont une "parole saine" ot une "condulte Irréprochable".

23 CPr. I Jn 5,6. Adversus haereses III 24,1.

24 A propos de $\Omega m 12,6, M_{0}-A$. Chevalliter défintt les charismata comme "les dons particuliors de la grace de Dieu, les dispensations personnelles, les "talents" remis a chacun par la générosité do Dieu, en vue d'un service à rendre à la communautén /op.cit., 146/ 
fonde sur la présence de l'Esprit qui est vérité.

De même qu'1l y a une relation Inclusive entre l'Eglise et l'Esprit qui est vérité et source de la connalssance de la vérité25, 11 y ane relation exclusive entre l'Eglise et l'erreur: "extra veritatem, extra Ecclesiam", dit Irénée. "Ceux qui sont en dehors de la vérité, c'est-a-dire ceux qui sont en dehors de l'Eglise"26.

Inversement, c'est de l'Eglise qu'il faut recevolr la vérité:

"Il ne Paut donc plus chercher auprès d'autres la vérité qu'il est facile de recevoir de l'Eglise, car les apotres, comme en un riche cellier, ont amassé en elle, de la fagon la plus plénière, tout ce qui a tratt à la vérité, afín que quiconque le désire y puise le breuvage de la vio. C'est elle, on effet, qui est la vole d'acces à la vie;"tous" les autres "sont des voleurs etsdes brigandsin. C'est pourquol 11 faut 1 es rejeter mals almer par contre avec un zéle extrême céríín 89 t de l'Eglise et saisir la Tradition de la

Alnsi 11 faut rejeter les hérétiques qui sont "étrangers à la vérité" ou encore "sophistes de mots plutôt que disciples de la véritén ${ }^{30}$, et Inversement recevolr la vérité de l'Eglise comme une transmission, la traditio veritatis, liée à la tradition apostolique qui se transmet avec la succession épiscopale.

Tradition apostolique, vérité et succession épiscopale sont 11ées, o'est pourquol Irénée, après avoir donné la liste des évêques de 1 'Eglise de Rome 31 ajoute:

"Vollà par quelle suite et quelle succession, la Tradition se trouvant dans l'Eglise, à partir des apó-

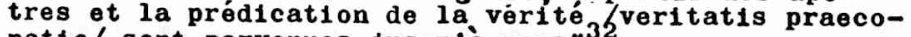
natio/ sont parvenues jusqu'a nous" 32 .

C'est dans l'Eglise qua lieu la traditio veritatis et la praeconatio veritatis, la vérité étant reque avant d'être annoncée et

..."Spiritu/s/ Dei qui praestat agnitionem veritatis". "L'Esprit de D1eu quil donne la connaissance de la véritén /IV 33,7 , SCh $100,818 \%$.

26 Adversus haereses IV 33,7, SCh 100, 817.

27 Jn 10,8.

28 Tt $3,10$.

29 Adversus haereses III $4,1, \operatorname{SCh} 211,45$.

30 Adversus haereses III 24,2 , SCh $211,475$.

31 Cfr Adversus haereses III $3,2-3$, SCh $211,32-38$.

32 Adversus haereses III 3,3 , SCh 211,39 . 
c'est par la succession des évêques que se transmet la tradition regue des Apôtres, à savolr qu'"1l n'y a qu'une seule et unique vérité \%unam et solam hanc veritatem/, celle-là même qui est transmise par 1 . Egl1sen33.

Enfin, par la succession ép1scopale, les évêques ou presby$\operatorname{tres}^{34}$, ne regoivent pas seulement la traditio veritatis ou encore la Regula veritat1s ${ }^{35}$, mass également le charisma veritatis certum:

"C'est pourquol 11 faut écouter les presbytres qu1 sont dans l'Eglise: 11 s sont les successeurs des apotres, alnsi que nous l'avons montré, et, avec la succession dans l'éplscopat, 11 s ont reçu ie sûr charssme de la vérité, selón le bon plaisir du Pó-

Après avoir montré la relation inolusive entre la présence des charismes, comme celui de "docteurs", dans l'Eglise et l'enselgnement de la vérité, et, d'autre part, la relation entre la tradition apostolique qui n'est autre que la tradition de la vérité ot la suocession épiscopale, 11 s'agit de se demander que signifie oette expression "sûr charisme de la vérité" liée à la succession épiscopale.

\section{II}

1. Entendu dans le sens objectif, le "charisma veritatis certumn37 est la doctrine apostolique de l'Eglise confiée à l'évêque

33 Adversus haereses III $3,4, \operatorname{SCh} 211,41$.

34 Sur l'équivalence des termes "presbytres" et "évêques", volr: J. Colson, Les fonctions ecclésiales aux deux premiers siecles, Paris 1956, 279, ot ldem, L'Eveque dans les communautés primit1ves, Paris 1951, 119.

35 Cfr Adversus haereses III 11, 1, SCh 211, 138-142.

36 Adversus haereses IV 26,2, SCh 100, 719.

37 Sur les différentes interprétations du "charisma veritatis certum" vo1r: $Y$. Congar, Mysterium Salut1s 15: Dogmat1que de 1'H1sto1re du Salut, Paris 1970, 210, note 73; P. Bacq, De l'Anoienne à la Nouvelie Alliance selon S. Irénée, Paris 1978, 202, note 2. 
au moment de l'ordination. Alnsi l'entendent $K$. Muller ${ }^{38}$, D. Van den Eynde $^{39}$, E. Molland ${ }^{40}$, A. Benoît $\hat{t}^{41}$ et H. Von Campenhausen ${ }^{42}$.

suller falt remarquer qu' "Irénée ne parle manifestement nulle part d'un charisme conctionnel /Amtscharisma/ particulier des évê-

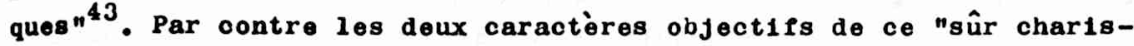
me de véritén sont

- la transmission de la vérité du kérygme par la succession aposto11 que,

- et la parole salne et la condulte Irréprochable de l'évêque.

Selon Van den Eynde, dans son livre sur "Les Normes de l'enselgnement ohrétien dans la littérature patristique des tro1s siocles", la tradition 44 Implique à la fols le "dépôt que l'Eglise a regu des apôtres" qu'Irénée nomme "kérygme de la véritén"45 ou "kérygme des apôtres" 46 , ou encore "tradition de la véritén"7 ou "tradition des apôtres"48, ce qui indique l'équivalence des termes "kérygmen et tradition - "paradosis", l'un soulignant le point de départ et l'autre la transmission, et la transmission elle-même de ce

38 K. Muller, "Das Charisma veritat1s" und das Eplskopat bei Irentus, ZNTW 23/1924/ 216-222.

39 D. Van Den Eynde, Les Normes de l'enselgnement chrétien dans la iltérature patristique de trols premiers siecles, Par1s 1933, 186-187.

40 E. Molland, "Le développement de l'idée de succession aposto11que", RHPR 34/1951/1-29; Irenaeus of Lugdunum and the Apostolic Succession, "The Journal of Ecclesiastical History" $1 / 1950$ / 12-28.

41 A. Benô̂t, L'apostolicité au second siècle, "Verbum Caro" 58 /1961/173-184.

42 H. Von Campenhausen, Kirchliches Amt und geistliche Vollmacht in den ersten dre1 Jahrhunderten, Tubingen 1953, 195.

43 K. Muller, op. cit., 219.

44 D, Van den Eynde définit la "tradition" chez Irénée comme "le depot que l'Eglise, a regu des apótres et qu'elle garde intact par la succession des évêques", op. cit., 163 .

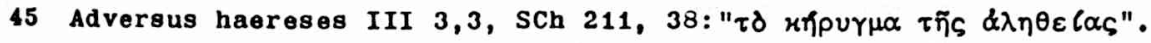

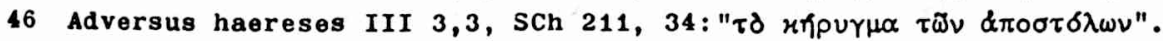

47 Adversus haereses III 4,1, SCh 211, 44: "tradit1o veritatis".

48 Adversus haereses III 3,3, SCh 211, 36: "apostolica Ecclesiae trad1tio"; 1b1dem, p.34: "trad1t10 ab Apostolis". 
dépôt par la succession éplscopale: kérygme/ripurua/ apostolique,

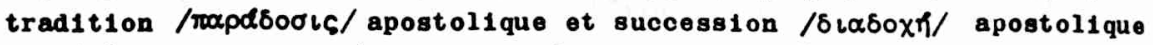
des évêques sont des élements lnséparablement liés car c'est l'apostolicité de l'Bglise qui est la preuve de sa vérité.

Par la succession apostolique les évêques regoivent donc à la fola lo kérygme de la vérité, la tradition de la vérité et lo sûr charisne de la vérité. Le sûr charisme de vérité est donc, conclut $D$. Van den Eynde, "identique à la doctrine des apôtres ot à la vérité elle$\rightarrow$ âmen 49 .

De même A. Benoît, dans son article sur "l'apostolicité au second sièclen", affirme que 10 "charisma veritatis certum" n'est pas "un charisme sacramentel regu par l'ordination et constituant une garantie surnaturelle de l'enselgnement apostolique authentiquen, comme Dom G. D1x le pense ${ }^{50}$, ma1s plutôt "la doctrine de l'Bglise confiée à l'évêque au moment de son ordination ${ }^{n 51}$.

La preuve a contrario c'est que, dans le même texte ${ }^{52}$, Irénée parle des presbytres qui sont devenus schismatiques et qui "s'éloignent de la succession authent1que /absistunt a principal1 successione/", et, par là, s'égarent de la vérité/decidunt a veritate/". Il ne s'aglt donc pas d'un don sacramentel, mals d'un charisme lié à la légitimité de la succession et à la eldélité de la doctrine.

2. Cependant l'interprétation objective du "charisma veritatis certum", l1é à la tradition et à la succession apostolique, ne rend pas compte du sens paulinien du terme "charisme". C'est pourquol Louis Ligier, dans son interprétation du "charisma veritatis certum" ${ }^{53}$ fait au contralre porter le poids de l'expression sur le "charisma" qui est un don de l'Esprit à l'Eglise.

Les réPèrences d'Irénée à I Cor 12,28 dans les deux textes précédement cités de III 24,1 et IV 26,5, Indiquent que, pour Irénée, "la

49 D. Van den Eynde, op. c1t., 187.

50 Dom G. Dix, Le ministère dans l'Eglise ancienne, Neuchâtel 1955, 45.

51 A. Benoit, op. c1t., 182.

52 Adversus haereses IV 26,2, SCh $100,718$.

53 L. Ligler, Le Charisma veritatis certum des Evêques, ses attaches liturgiques, patristiques et bibliques, in: L'homme devant D1ou. Mélanges II. de Lubac, Par1s 1964, I, 247-268. 
théologie de la succession apostolique et celle des charismes de l'Esprit s'unissent 1c1 l'une à l'autron54.

Charismes et institution bien loin de s'opposer sont 11és: 11 y a une "structure charismatique de l'Eglise" où "Dieu a établi des apôtres, des prophètes et des docteurs et toute autre opération de l'Esprit" 55 , et lo "charisma veritatis certum" est "pour l'évêque successeur des apôtres, un don propre qui ne garantit la valeur de son enselgnement qu'une fols assurée l'authenticité de sa succession apostol1quen 56 .

La succession épiscopale est une garantie de la valeur de l'enselgnement, mals l'élection à l'épiscopat est un don gratuit de Dieu, Indiqué par l'expression "selon le bon plassir du Père/secundum placitum Patris/". "L'élection et la consécration d'un évêque, dit Ligier, sont bien un geste du bon plaisir du Père. De sa grâce viennent le sacerdoce et la charge pastorale: l'effusion gratuite de l'Esprit souverain les confèrent" 57 .

Le caractère personnel du charisme de vérité reçu par l'évêque au moment de l'ordination est montré par R.P.C. Hanson ${ }^{58}$, à la suite de E. Flesseman-Van Leer ${ }^{59}$, qui insiste sur la part de Dieu dans la désignation des ministres ot sur le fait que la consécration épiscopale est précédée et accompagnée par des dons spirituels accordés aux évêques et enfin par N. Brox qui souligne l'aspect humain du charisme qui est une "qualité de l'homme, produite par l'Esprit, on vue de l'accomplissement du plan de salut dans l'Egl1se"60.

\section{III}

En fin de compte, si l'on porte l'accent sur la vérité et la certitude dans l'expression "charisma veritatis certum", on est oonduit à l'identifier à la vérité elle-même et au contenu objectif de

54 L. Ligier, op. c1t., 249.

55 Adversus haereses III 24,1, SCh 211, 473.

56 L. Ligier, op. cit., 248.

57 Ibidem, 260.

58 R.P.C. Hanson, Tradition in the Early Church, London 1962, 159-160.

59 E. Flesseman-Van Leer, Tradition and Scripture in the Early Church, Assen 1954, 119-122.

60 N. Brox, "Charisma veritatis certum/Zu Irendus Adv. Haer. IV $26,2 /$ ", ZKG 75/1964/331. 
la doctrine apostolique qui transmse par la succession des évêques ridèles à cette doctrine.

S1 l'on accentue, au contralre, le charisme comme don de l'Esprit, accordé selon "le bon pla1sir du Père", à tel évêque, dans la succession éplscopale, on est conduit à accentuer le caractère personnel de ce don.

Dans un cas, 11 s'aglt du don de la vérité elle-même, dans l'autre, du don d'enselgner ou d'annoncer cette vérité, dans le premier, de la doctrize ou de la tradition apostolique, dans le second des "docteurs" que l'Esprit Saint a placés dans l'Eglise.

Les aspects du "charisma" et de la "veritas" sont inséparables comme la Parole et l'Esprit, la vérité et l'intelligence de cette vérité. Or ce sur quol porte le "sûr charisme de vérité" c'est l'intelligence des Ecritures, d'après la doctrine des apôtres.

Personne n'a souligné jusqu'ici le contexte immédiat de ce passage du Livre IV sur le "charisma veritatis certum". Il ne s'agit pas 1c1, comme au Livre III, de la Tradition apostolique, mais de l'interprétation des Ecritures. Ce qu'Irénée veut montrer c'est que l'interprétation des Ecritures se fait dans la relation maître-disciple qui est une relation au Christ ressuscité dans l'Eglise:

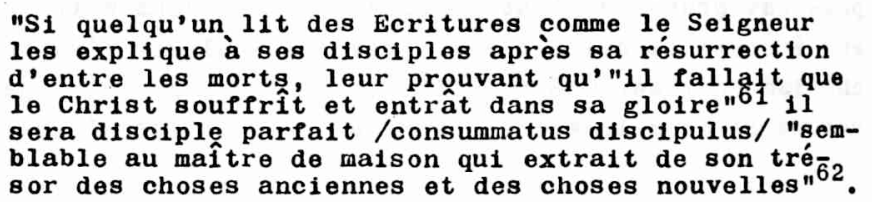

Ft tout de suite après, Irénée parle des"presbytres qui sont dans l'Eglise et qui, avec la succession apostolique, ont regu le sûr charisme de vérité"63, car 11 s sont ces "disciples parfaits" qui extraient du trésor des Ecritures et de la Tradition apostolique qu'ils ont regu en héritage avec la succession apostolique ${ }^{64}$, les nova et veterá, grâce au "conse1l de l'Esprit" 65 .

61 Lc 24,47 .

62 Nit 13,52 - Ádversus haereses IV 26,1, SCh 100, 717.

63 Adversus haereses IV 26,2, SCh 100, 718.

64 A. Benoît a bien montré qu' "au second siècle la notion d'apostolicité va se lier à quatre termes qui reçoivent le qualificatif d'apostolique: ce sont l'Ecriture apostolique, la Tradition apostolique, la Succession apostolique et le Symbole apostolique"/op. c1t $176 \%$ Ces quatre termes sont également liés dans le charisma veritatis certum.

i5 Adversus haereses IV 27, 1, SCh 100, 728-730. 
De même, "Si quelqu'un crolt au seul Dieu qui a falt toutes choses par son Verbe... toute parole/sermo/ émanée de lui aura sa consistance, pourvu qu'il lise aussi les Ecritures d'une maniére attentive auprès des presbytres qui sont dans l'Eglise; puisque c'est aupres d'eux que se trouve la doctrine des apôtres" 66

On retrouve la structure 'Ubi-ib1' dans cette phrase:

"apud eos qui in Ecclesia sint presbyteri

apud quos est Apostolica doctrina"

Auprès des presbytres qui sont dans l'Eglise, là se trouve la dootrine des apôtres.

Il y a donc une double relation de mâtre à disciple, relation des presbytres, disciples parfaits, et du Christ, et relation de ceux qui lisent les Ecritures et des presbytres, auprès de qui se trouve la doctrine des apôtres. Relation des presbytres au christ, à travers la succession apostolique et l'imitatio Christi, et relation des lectenrs de l'Ecriture aux presbytres de l'Eglise, auprès de qui se trouve la doctrine apostolique.

Le "sûr charisme de vérité" porte donc avant tout sur l'interprétation des Ecritures comme le montre l'inclusion de toute la seotion sur l'enseignement des presbytres/IV 26,1 - IV 32,1/ entre les deux phrases cond1tionnelles: "S1 quis legat"... "S1 autem credat qu1s"...

Doctrine des apôtres /doctrina apostolica/ ou tradition apostolique et 1nterprétation des Ecritures sont liées dans le "charisma veritatis certum", de telle sorte que la lecture des Ecritures ne peut se raire qu'en Eglise /1n Ecclesia/.

Mais le "charisma veritatis certum" s'étend également à tous les temps ot à tous les lieux:

"Un tel disciple, dit Irénée, vraiment "spirituel" - pour avoir regu l'Esprit de Dieu qui fut depuis le commencement avec les hommes dans toutes les "économies" de Dieu, prédisant l'avenir, montrant le présent et racontant le passé - "Juge de tous les hommes et n'est Jugé par personne"67.

c'est pourquol le presbytre, disciple parfalt, exerce le "sûr charisme de vérité" qu'il a reģu avec la succession épiscopale dans le "jugement" des hérétiques ou le discernement entre la doctrine de

66 Adversus haereses IV $32,1, \operatorname{SCh} 100,799$.

67 Adversus haereses IV 33, 1, SCh 100, 803. - I Cor 2,15. 
Marcion ${ }^{68}$, Valentin ${ }^{69}$, des faux prophètes 70 ou des rauteurs de sohismes 71 et la doctrine apostollque, ou entre la "gnose orgue1lleuse" et la "gnose vraie"/agnitio vera/72.

Il juge de tous les hommes et n'est jugé par personne, selon I Cor 2,15, car 11 a une fol intégrale/P1des Integra/ dans lo D1ou tout-puissant, une adhésion ferme /assensio f1rma/ dans le F1ls de Diou, N.S.J.C., et, par l'Esprit de Dieu, une connalssance de la vérité /agnitio veritat1s/, o'est-à-dire des néconomies du Père et du Fils /dispositiones Patris et Filil/, selon chaque génération, on vue des hommes, comme le vout le Pèren"73.

C'est donc la confession de fol trinitalre on Dieu, Père, Pils et Esprit qui fait Ie disciple parfalt et fonde le charisme de vérité, charisme de l'Esprit, donné aux presbytres, dans l'Eglise, par la succession épiscopale depuis les apôtres du Christ, selon lo bon plaisir du Pòre.

Ysabel de Andia CNRS, Paris

68 Adversus haereses IV $33,2, \operatorname{SCh} 100,804$.
69 Adversus haereses IV $33,3, \operatorname{SCh} 100,808$.
70 Adversus haereses IV $33,6, \operatorname{SCh} 100,814$.
71 Adversus haereses IV $33,7, \operatorname{SCh} 100,816$.
72 Adversus haereses IV $33,8, \operatorname{SCh} 100,818$.
73 Adversus haereses IV $33,7, \operatorname{SCh} 100,819$. 